\title{
Alter
}

Revue de phénoménologie

26 | 2018

La nature

\section{Réflexions sur l'espace et le temps dans la phénoménologie de Renaud Barbaras}

Aurélien Deudon

\section{(2) OpenEdition}

1 Journals

Édition électronique

URL : http://journals.openedition.org/alter/645

ISSN : 2558-7927

Éditeur :

Association ALTER, Archives Husserl (CNRS-UMR 8547)

Édition imprimée

Date de publication : 31 décembre 2018

Pagination : 197-212

ISBN : 978-2-9550449-4-0

ISSN : 1249-8947

\section{Référence électronique}

Aurélien Deudon, «Réflexions sur l'espace et le temps dans la phénoménologie de Renaud Barbaras », Alter [En ligne], 26 | 2018, mis en ligne le 31 décembre 2019, consulté le 22 mars 2020. URL : http:// journals.openedition.org/alter/645 ; DOI : https://doi.org/10.4000/alter.645 


\section{RÉFLEXIONS SUR L'ESPACE ET LE TEMPS DANS LA PHÉNOMÉNOLOGIE DE RENAUD BARBARAS}

Aurélien Deudon

Alors que la conception habituelle de l'espace et du temps insiste sur la différence insurmontable de leur nature, sur leur incommensurabilité malgré leur coexistence (la réalité se donnant simultanément dans l'espace et le temps), la conception philosophique à laquelle nous voudrions faire droit ici revendique leur articulation. Envisagée phénoménologiquement, cette question de l'articulation ne contrevient pas seulement à l'idée courante selon laquelle l'espace n'a rien à voir avec le temps, mais elle neutralise également l'attitude qui tenterait de subsumer leur intrication sous le paradigme de l'interaction causale : en effet, l'épochè husserlienne met entre parenthèses l'existence en soi du monde entendu comme unique réalité spatio-temporelle et le paradigme causaliste qui régit les interactions des objets qui la peuplent - d'ailleurs, même hors épochè, la généralisation de la causalité ne va pas du tout de soi, car le tiret qui soude la locution «spatio-temporelle » ne désigne pas l'interaction entre deux objets mais la jointure entre les deux dimensions au sein desquelles se livrent les objets eux-mêmes. Dans tous les cas, au sein du cadre fondationaliste de la phénoménologie, la question de l'intrication de l'espace et du temps va être traitée dans une perspective résolument génétique. Finalement, outre l'interrogation sur la nature véritable de ces deux dimensions, se demander comment l'espace peut s'articuler au temps revient à demander si l'un a la primauté dans le conditionnement de l'autre ou bien s'ils se trouvent dans une situation de conditionnement mutuel. Ces précisions apportées, nous voudrions faire droit à l'articulation inédite que thématise la phénoménologie de Renaud Barbaras en explicitant la phrase directrice suivante : «Elle [la temporalité] est ce qu'il arrive à un monde (c'est-à-dire à une vie) éternel dont l'exis- 
tence n'est que celle de l'espace, lorsqu'il se perd lui-même en luimême, se détache de lui-même et se donne ainsi à lui-même ${ }^{1}$. Au travers de cette affirmation on pressent d'ores et déjà qu'au sein de cette phénoménologie se joue l'avènement d'une nouvelle pensée de l'espace et du temps. Prendre la mesure de cette originalité nécessite deux moments : d'une part, restituer le cadre phénoménologique emmi duquel elle prend sens et consistance ; d'autre part, déplier et prolonger ce que les assertions programmatiques et embryonnaires de ce système recèlent sans évoquer - évidemment un tel prolongement se risquera à forer des ramifications théoriques absentes du cadre matriciel barbarassien.

\section{Les contraintes de la corrélation originaire}

Toute entreprise phénoménologique se voue à expliciter ce que Husserl nommait $\mathrm{l}^{\prime}$ «a priori universel de la corrélation $»^{2}$, que cette corrélation soit celle de la conscience et de l'objet ou pour la phénoménologie post-husserlienne celle plus fondamentale du sujet (quelles que soient ses ententes : Dasein, néant, chair, désir, etc.) et du monde. La singularité de ce fondement corrélationnel c'est qu'il désigne une relation interne et non externe. En effet, il ne s'agit pas d'une relation s'instaurant après coup, entre deux termes préexistants comme pourrait l'entrevoir un réalisme naïf : l'extériorité des termes corrélés impliquerait alors la contingence de leur corrélation et celle-ci ne pourrait en rien constituer un fondement, un a priori. Il ne s'agit pas non plus de considérer qu'un terme constituerait l'autre comme peut l'entendre l'idéalisme ou un certain réalisme scientifique - ce serait en effet la complexité de certaines organisations matérielles qui engendrerait le vivant humain - car alors la corrélation serait à ce point intériorisée qu'elle se nierait en tant que mise en rapport de deux instances réellement distinctes, elle nierait son essence corrélationnelle comme telle. En réalité, la corrélation phénoménologique exige de penser une relation qui précède et fonde les termes simultanément : la relation première engendre les termes, leur sens d'être. C'est parce qu'elle évite de dévier autant par excès d'extériorité, vers une relation contingente qui a pour paradigme la rencontre empirique, que, par défaut d'extériorité, vers une intériorisation sans relation (une consti-

\footnotetext{
${ }^{1}$ R. Barbaras, Dynamique de la manifestation, Paris, Vrin, 2013, p. 327. Noté désormais DM.

2 E. Husserl, La crise des sciences européennes et la phénoménologie transcendantale, Paris, Gallimard, 1976, § 48.
} 
tution), que la phénoménologie peut exhiber le caractère essentiel de la corrélation du sujet et du monde et en faire l'a priori strict sur lequel repose son discours. Ajoutons que pour la phénoméno-logie le tissu conjonctif qui engendre les instances distinctes de l'apparaissant et du destinataire c'est naturellement le phénomène, l'apparition. Quoi qu'il en soit, ce fondement corrélationnel délivre et exige le respect de deux contraintes paradoxales : d'un côté, penser la corrélation de deux termes supposent de ne pas les identifier, car l'identité ne supporte pas l'écart d'une mise en relation. Il faut donc reconnaître que les deux termes seront différents l'un de l'autre: moins formellement, que le sujet n'est pas le monde et inversement. Cependant, d'un autre côté, et plus profondément sans doute, quelle que soit la nature de la corrélation, celle-ci suppose également une communauté entre les termes qu'elle relie : il est évident qu'à défaut de cette parenté les termes ne pourraient même pas être corrélés et se rateraient par principe - même dans une relation d'opposition, les opposés supposent une même nature, un ordre commun (ex: « petit » et " grand » s'opposent dans l'ordre de la taille). Moins formellement, il faut donc reconnaître que le sujet a à voir avec le monde, il partage nécessairement quelque chose avec lui. En somme, les termes de cette corrélation essentielle sont à la fois différents et apparentés, autre et même. Et puisque dans une véritable corrélation la différence des corrélés, quelle que soit son amplitude, ne vient jamais anéantir leur communauté il faut sans doute dire que la différence se prélève, se détache d'une communauté primitive, que l'autre se donne toujours sur fond de même, et peut-être même que les corrélés sont authentiquement autres parce que profondément mêmes ${ }^{3}$.

\section{Dynamique du sujet et du monde}

La phénoménologie qui a relevé le plus clairement cette double contrainte initiale de l'a priori corrélationnel et qui s'est évertuée à déterminer un être du sujet en congruence avec ce cadre apparemment paradoxal ${ }^{4}$, c'est celle de Barbaras. Tandis que les grandes phénoménologies antérieures ont généralement mis en avant une contrainte plus que l'autre - par suite elles ont tantôt déterminé le sujet comme

\footnotetext{
${ }^{3}$ Le cadre phénoménologique que nous nous apprêtons à restituer permettrait de traduire ces rapports de l'autre et du même en termes d'être, de mouvement et d'immobilité, et ainsi de rejouer concrètement la dialectique (abstraite) des grands genres que propose Platon dans son Sophiste.

${ }^{4}$ Nous verrons qu'en réalité le paradoxe n'existe que pour un point de vue statique.
} 
« être-au-monde », « néant », etc., si elles se polarisaient sur la différence ; tantôt comme "chair» si elles revendiquaient la communauté -, la phénoménologie barbarassienne prend à bras le corps les deux contraintes et, corroborant la perspective de Jan Patočka, propose de saisir le sujet comme mouvement ${ }^{5}$. Contre le réductionnisme de la physique moderne, la phénoménologie dynamique vise à montrer que le mouvement du sujet n'est pas un simple déplacement pour deux raisons majeures. D'abord parce que le mouvement purement local ne met jamais en jeu l'être du mobile, du déplacé : l'être du mobile est toujours préalablement posé comme substrat statique et immuable et son déplacement n'est qu'un accident. De son côté, le sujet n'est pas en mouvement comme le mobile, au contraire, il est mouvement au sens où il ne préexiste pas au mouvement mais s'accomplit tout entier en son mouvement - il en est moins la source que le terme. À la différence du simple déplacement, le mouvement subjectif est donc accomplissement, il met en jeu l'être du sujet au sens où le mû devient lui-même au fur et à mesure de son mouvoir. D'autre part, le pur mouvement local est aveugle, il ne conditionne aucune apparition alors que le mouvement du sujet est phénoménalisant, fait apparaître, éclaire ce qui se trouve sur son chemin. Comme le dit à plusieurs reprises Barbaras, ce mouvement subjectif est donc plus qu'un déplacement et moins qu'une représentation, car il articule essentiellement une avancée dans le monde et une ouverture au monde, c'est-à-dire un éclairage du monde. On voit donc que la détermination du sujet comme mouvement conjoint une dimension de différence - le sujet va vers le monde, y aspire ou le désire en l'ouvrant parce qu'il ne l'est pas - et une dimension d'appartenance - le sujet est dans le monde comme tout étant, et, en tant que mouvement, il s'appuie sur le monde comme sur son sol. Mais Barbaras d'ajouter que si le sujet peut être à la fois dans le monde (comme tout étant) et condition de la co-apparition du monde (cosmophanie) en chaque étant c'est en raison d'une parenté plus profonde qu'une simple parenté ontique (celle d'inclusion, seraitce dynamique, de l'étant au sein du monde), d'une parenté qu'on doit dire ontologique et qui exhibe une hyper-appartenance du sujet au monde. Il y a donc simultanément deux niveaux d'appartenance du sujet au monde : le sujet, comme tout étant est dans le monde comme une partie dans son tout, mais en tant que sujet il ne peut ouvrir au monde que s'il est $d u$ monde et lui hyper-appartient. L'appartenance signifie sa communauté avec les étants du monde, l'hyper-appartenance signifie sa communauté ontologique avec le monde.

${ }^{5} \mathrm{DM}$, Première partie, chapitre 2. 
Évidemment ces considérations sur la parenté ontologique entre le sujet et le monde vont permettre à la phénoménologie dynamique de se dépasser vers une cosmologie entendue comme dynamique phénoménologique. En effet, en vertu de l'a priori métonymique ou méréologique, si le sujet est bien une (hyper-)partie du tout, du monde, alors la partie est nécessairement de la même nature que le tout. Par conséquent, puisque nous connaissons la nature du sujet nous pouvons inférer la nature du monde : si le mode d'être du sujet est bien dynamique alors, en vertu de la parenté partie-tout, le mode d'être du monde est également dynamique. Bien sûr, le monde étant saisi désormais comme mouvement, il faut prendre au sérieux sa parenté avec le sujet et signaler que son mouvement n'est pas non plus un pur déplacement, mais bien un mouvement d'accomplissement - par lequel le monde se fait monde, devient lui-même, se mondifie - et un mouvement de phénoménalisation. Au-delà de cette parenté dynamique, le mouvement cosmique n'a somme toute pas grand chose à voir avec le mouvement subjectif et c'est pourquoi il faut parvenir à déterminer en quel sens le monde phénoménalise, fait apparaître les choses. Suivant à nouveau les analyses patočkiennes, Barbaras montre que ce mouvement de mondification phénoménalise, fait apparaître, en produisant matériellement les étants par différenciation. Faire apparaître correspond alors à l'œuvre d'un mouvement qui fait sortir de l'indistinction en assignant des limites, des frontières (finis) : le mouvement du monde est un mouvement ontogénétique qui procède par définitions continues (et non par création, surgissement ex-nihilo) : la genèse de l'étant est délimitante. Les étants délimités par la puissance du monde sont d'ailleurs nécessairement pris dans cette puissance qu'il faut saisir comme un fond indéterminé se différenciant incessamment. Dès lors, les étants ne sont jamais pleinement des individus au sens strict, mais sont toujours en voie $d^{\prime}$ individuation ou de désindividuation en ce que l'indétermination du fond les transit de part en part et finit toujours par les engloutir. Le déterminé est précaire, l'indéterminé est pérenne. Concrètement, le mouvement du monde s'actualise à travers tous les mouvements mondains qui eux-mêmes circonscrivent des étants : le mouvement mondain se finitise donc sans cesse à travers des mouvements intramondains (le monde n'est pas autre chose que son contenu) qui font autant qu'ils défont les étants. Le monde correspond donc à une nature processuelle, à une phusis par laquelle tout individu n'advient que par provision. 


\section{Puissance subjective et surpuissance phusique}

Chemin faisant, on remarque que la différence entre le mouvement subjectif et le mouvement mondain est une différence de puissance : alors que le mouvement mondain jouit d'une puissance insigne lui permettant de faire tout ce qu'il peut faire, de produire matériellement les étants ; le mouvement subjectif, lui, se caractérise par une impuissance constitutive en ce qu'il ne peut rien produire (si ce n'est des gestes) mais peut seulement faire paraître. Notre faire apparaître est un faire paraître qui n'a pas la puissance suffisante pour faire être : ainsi le sujet aspire au monde à défaut d'avoir la puissance ontogénétique de le produire - le sujet est donc fondamentalement mouvement désirant : le véritable désir n'aspirant qu'à devenir et rejoindre un mouvement supérieur. Mais l'impuissance est toujours l'envers d'une puissance. En effet, la puissance propre du sujet consiste justement à faire paraître les étants en tant que tels, c'est-à-dire à prolonger l'œuvre de distinction du mouvement mondifiant en déracinant les étants de leur fond d'indétermination, en les détachant du monde qui les produit. Le sujet est donc un mouvement qui a la puissance de poursuivre, à sa façon, formellement et non matériellement, la définition et la formation d'unités, d'individus en séparant davantage le producteur de ses produits, le mondifiant du mondifié. Et puisque le sujet ne cesse de repousser le monde "derrière » les étants qu'il fait saillir, son mouvement vers le monde se trouve inexorablement exacerbé et relancé par chaque apparition. Cette insatiabilité du mouvement, caractéristique du désir, est tout autant une dimension de sa puissance. En somme, la puissance du sujet réside dans sa capacité à poursuivre indéfiniment l'œuvre délimitante du monde. Dans cette perspective, on voit que l'apparaître secondaire/subjectif se place dans la droite ligne de l'apparaître primaire/mondain car il s'explicite en termes bergsoniens de découpages, de délimitations : c'est en ce sens aussi que Barbaras après Patočka peut expliquer que la synthèse subjective repasse sur les pointillés de la synthèse matérielle ${ }^{6}$. D'ailleurs, puisque phusis et sujet opèrent tous deux fondamentalement par différenciation ou définition pour faire apparaître, on doit avouer que tout mouvement est originairement ciselant, ontologiquement tranchant. Cependant, dans ce cadre, que devient l'autre grand type d'apparaître, de désoccultation sur lequel s'axe la philosophie traditionnelle? Que devient l'apparaître dévoilant vis-à-vis de l'apparaître différenciant?

${ }^{6} \mathrm{DM}$, Deuxième partie, chapitre 4, p. 214 et $s q$. 
Quel type de rapport entretiennent-ils? On voit déjà que leur différence ne recouvre pas la différence de l'objectif et du subjectif puisqu'on vient de voir que le découpage, le détachage est aussi une œuvre subjective. En revanche, on peut vite s'apercevoir que le dévoilement est un apparaître exclusivement subjectif - seul un sujet peut ôter un voile. De plus, le dévoilement dépend éminemment de la différenciation. En effet, c'est parce que le sujet prolonge les unités esquissées par la phusis, différencie à nouveau ce qui a été proto-différencié du fond indéterminé du monde - le mouvement subjectif porte la différenciation à la seconde puissance - que justement ce fond est nettement repoussé derrière l'unité de l'étant, que le monde comme tel se voile dans l'apparition. C'est donc parce que le mouvement subjectif différencie des étants et par là voile le monde d'où proviennent ces étants, qu'il peut en un second temps vouloir dévoiler le monde, accéder à ce qui recule abyssalement à chaque définition subjective. Autrement dit, puisqu' on ne peut soulever qu'un voile préalablement apparu, délimité, il faut conclure que le dévoilement est toujours second et présuppose toujours la constitution, la différenciation préalable d'un voile - le sujet ôte nécessairement le voile qu'il a lui-même installé. Dès lors, quoiqu'en dise Heidegger, le voilement est toujours le fait d'un sujet de sorte que faire du voilement l'essence même de l'être paraît être un reliquat fâcheusement subjectiviste. En somme, le dévoilement (la logique du secret) est nécessairement le dernier apparaître, il est nécessairement subordonné à l'apparaître différenciant comme à sa condition d'apparition.

Après avoir repéré l'envers de l'impuissance du sujet, on serait tenter de dire que la puissance du procès mondain a elle aussi pour envers une impuissance qui correspondrait à l'inachèvement constitutif qu'implique le devenir-monde du monde : le monde ne cessant de s'accomplir a l'imperfection et donc l'impuissance de ne jamais être pleinement. Mais on le perçoit vite, ce raisonnement demeure foncièrement attaché à l'idéal parménidien de l'immobilité car il assimile la mobilité et le devenir-soi-même à une impuissance qui aurait sa vérité dans le devenu-soi-même statique, la plénitude de l'immobilité. En réalité, rien ne pourrait accomplir le monde, ce dernier ne tend vers rien, vers aucune figure idéale - au passage cela reviendrait à le subjectiviser - de sorte que son inachèvement n'est en rien une impuissance mais désigne au contraire l'excès inentamable de la puissance sur ses œuvres. C'est pourquoi l'inachèvement du monde est ipso facto son achèvement : on pourrait dire que le procès mondain est un achèvement sans fin car c'est en tant qu'accomplissement que le monde est accompli. De plus, si le monde pouvait atteindre une figure stable, 
immobile, il renoncerait à toute sa puissance car une vraie puissance se met nécessairement en œuvre, et donc (se) mobilise : l'immobile est ce qui ne peut rien, l'achevé est ce qui ne peut plus, est l'épuisé, le mort. Enfin si le monde possède un mode d'être dynamique c'est que son mouvement est inépuisable : en effet, si le mouvement mondain était transitoire, provisoire, la vérité ontologique du monde serait l'immobilité et non la mobilité de telle sorte que la communauté ontologique avec le sujet serait contredite et désavouée. Il faut donc conclure que la puissance du procès mondain est inépuisable, intarissable et que cette puissance mondifiante sans trace d'impuissance doit être qualifiée de surpuissance. Barbaras insiste lui-même à plusieurs reprises sur le fait que le mouvement mondain coïncide avec la mise en œuvre d'une puissance qui se nourrit de ce qu'elle engendre, qui s'accroît de ce qu'elle réalise de sorte que sa dépense ne fait pas alternative avec sa régénération voire son amplification. Dans cette logique de prodigalité cronophage ${ }^{7}$, la puissance phusique a une réserve qui ne peut s'essouffler, et est bien surpuissance.

\section{Spatio-atemporalité de la phusis}

Au niveau de cette surpuissance mondifiante se pose alors la question de l'espace et du temps. On l'a dit le mouvement du monde différencie matériellement et produit ainsi une multiplicité primitive, $c^{\prime}$ està-dire à la fois une multiplicité d'étants qui n'est pas que numérique, mais avant tout qualitative ; et une multiplicité dominée par l'unité, par le fond de puissance indifférenciée, donc une pluralisation inchoative, non pluralisée. Dès lors la cosmogenèse est un procès d'individuations et par là d'attribution de sites à l'étant dans la mesure où la circonscription engage nécessairement l'apparition d'un site ${ }^{8}$. On doit

\footnotetext{
${ }^{7}$ Par le néologisme «cronophage» nous voulons qualifier la logique propre à la puissance telle qu'elle a été cernée par le mythe grec de Cronos - il ne faut évidemment pas confondre ce terme avec son homophone «chronophage » qui fait référence à un temps que la phusis ignore comme nous allons le voir. En effet, en mettant en scène un dieu archaïque, un titan ayant pour caractéristique principale de se nourrir de ses propres enfants, le mythe capte, à nos yeux, la vérité profonde de l'être de la puissance. D'une manière générale, les grands mythes intuitionnent toujours des vérités ontologiques et métaphysiques en les formulant sous la forme d'histoires, en les ontifiant au gré d'une narration. Dès lors, il revient au lecteur averti ni de croire en l'existence réelle de telles histoires et en l'axiologie qu'elles mettent en scène, ni de reléguer de telles histoires au compte des fadaises et des fantaisies de l'esprit humain. Car, par-delà la crédulité et le scepticisme, il s'agit de repérer et d'accéder à l'intuition séminale et révélatrice qui anime chaque épisode mythique. Le mythe peut alors acquérir un double rôle: soit son intuition vient confirmer, lester le propos philosophique ; soit il vient indiquer une voie que la philosophie se doit d'inspecter.

${ }^{8}$ R. Barbaras, L'ouverture du monde, Paris, Édition de la Transparence, 2011, p. 229.
} 
donc reconnaître que cette surpuissance phusique implique un espace archaïque aux deux sens du terme : d'un part, cet espace commence, $c^{\prime}$ est le premier espace; d'autre part cet espace commande tous les autres de sorte que toute spatialité dépendra et dérivera de cette spatialité comme de sa source. On comprend par là que la phusis est un mouvement qui ne se déploie pas dans un espace préalablement ouvert et déployé, mais au contraire est un mouvement qui déploie et ouvre l'espace, qui spatialise, qui ébauche l'extériorité des étants en différenciant leur site. Ce ne sont donc pas les étants qui se meuvent dans l'espace c'est la spatialisation (l'assignation d'un site) qui différencie les étants en les mobilisant. Et si le mouvement mondain n'a pas de fin et qu'aucun étant $n^{\prime}$ accède à une individuation définitive, alors il faut dire que la spatialisation est incessante, toujours recommencée et qu'aucun espace du monde ne refroidit, ne tombe dans la fixité, l'immobilité de la pure extériorité. Il faut encore remarquer que cet espace de gestation (et corrélativement de disparition) des " choses », cette spatialité archaïque possède deux grandes caractéristiques : d'un côté, en tant qu'il ne s'agit pas tant d'un espace en mouvement que d'un espace-mouvement (car il appartient à la nature même du mouvement mondain de se spatialiser), cet espace est nécessairement fluide et instable. D'un autre côté, en tant que les étants situés ne sont pas pleinement extérieurs les uns des autres, que l'indistinction ne cesse de les traverser, et qu'aucun vide n'aère ce procès de définition, il faut avouer qu'il s'agit d'un espace compact, sans véritable distance de sorte qu'il faudrait parler de distan-ciment entre les sites pour souligner que la différenciation phusique, l'ajointement cosmique conjoint pour autant qu'il disjoint, cimente pour autant qu'il distend. En ce sens, la massivité de la phusis renvoie à une espèce d'horror vacui constitutive de la nature. Par conséquent, la spatialisation du monde correspond à un espace naissant fluide et dense, à une spatialité élastique dont chaque élongation différenciante est aussi un point de compacité. Avec cette spatialisation pluralisante on serait tenter d'affirmer que c'est à ce niveau anonyme, sans sujet que se manifeste la célèbre " déflagration de l'être » dont parle Merleau-Ponty dans L'CEil et l'Esprit, mais il faudrait d'emblée compléter l'expression en rappelant que cette déflagration cosmique est toujours aussi « inflagration », que l'explosion est solidairement implosion dans la mesure où la pluralisation demeure inchoative, prise dans le ventre compact du monde.

Dans un second temps, il faut rappeler que le mouvement phusique est un mouvement qui va du monde vers le monde, qui différencie certains étants autant qu'il en indifférencie d'autres puisque le fond indifférencié, duquel le monde tire sa surpuissance, est cette réserve de 
puissance qui perdure toujours au sein de ce qui s'y différencie. Dès lors, il faut conclure que le mouvement du monde n'implique aucun avant, ni après, aucun passé ni avenir car, comme le dit Barbaras ${ }^{9}$, le monde est toujours déjà passé et jamais passé. En fait, passé et avenir passent l'un dans l'autre puisque le fond indifférencié (le passé) persiste dans sa différenciation (l'avenir) - d'ailleurs le différencié est toujours provisoire et plongera tôt ou tard dans l'indifférencié au profit d'une autre différenciation. On peut donc tout autant dire que le passé « poursuit » ou colle l'avenir jusqu'à le reprendre et à devenir son avenir, et symétriquement que l'avenir est toujours déjà au passé, dépassé par son propre passé. Si antérieur et postérieur ne cessent de s'échanger - la présence correspond au jeu de cet échange -, et perdent leur pertinence catégoriale ici, $c^{\prime}$ est que le procès mondain est un devenir sans successivité unilatérale, sans progrès. Ce devenir qui piétine ne relève pas de la temporalité et n'est donc pas non plus dans le temps (on verra que le continuum objectif du temps suppose une temporalisation originaire). Pour autant, dire que le mouvement mondain n'est pas temporel ce n'est pas dire qu'il est intemporel. Il faut plutôt avouer qu'il est atemporel - neutre plutôt qu'opposé à la temporalité ou pré-temporel dans la mesure où l'on verra que c'est le mouvement spatialisant du monde qui est la co-condition de la temporalité. En ce sens catégoriser la phusis selon les dimensions temporelles n'est certes pas pertinent puisqu'elle échappe à la temporalité ; mais ce n'est pas pour autant une projection indue du sujet sur un être absolument étranger au temps puisque qu'il le possibilise. On n'échappe au temps qu'en étant sur son seuil. La phusis est une temporalité nulle et pourtant avenue ou plutôt pouvant venir. Même si Barbaras confirme ce caractère pré-temporel du procès mondain dans la mesure où « [1]a physis est sans temps mais se prête à la temporalité »10, on peut pourtant relever certaines formules qui contreviennent à ce caractère. Par exemple, dans Dynamique de la Manifestation ${ }^{11}$, il en vient à conclure : « le monde, quoique dynamique, est étranger au temps ». Or, on voit que s'il était réellement étranger, c' est-à-dire radicalement autre que le temps il serait incapable d'en conditionner l'apparition. Au contraire nous voudrions montrer que loin d'être étranger voire allergique au surgissement du temps, l'être dynamique en est la condition sine qua non - certes condition à elle seule insuffisante sinon le monde serait déjà temporel. La temporalité, en tant que telle, n'apparaîtra qu'avec

\footnotetext{
${ }_{9}^{9}$ R. Barbaras, Métaphysique du sentiment, Paris, Cerf, 2016, p. 92-93.

${ }^{10}$ DM, p. 188.

${ }^{11}$ DM, p. 328.
} 
ces étants qui ne sont pas de simples productions du monde et qu'on nomme les sujets vivants.

Enfin, puisque le mouvement n'est en rien un état passager du monde, mais ne désigne rien de moins que son mode d'être, il faut conclure que le mouvement surpuissant de la phusis ne peut ni avoir commencé, ni se finir. Certes, cela contreviendrait à la nature foncièrement dynamique du monde d'avoir été immobile ou de devenir immobile ; mais si, imaginant aller plus loin, on postulait un être d'avant le monde ou un être après le monde qui serait incommensurable à notre monde on renoncerait aussi à la détermination du monde comme totalité omni-englobante. En effet, si le monde est réellement ce tout absolu, rien ne peut être hors de lui, avant ou après lui : un tout absolu est nécessairement exclusif, et qu'il y ait deux touts absolus serait contradictoire car aucun des deux ne totaliserait véritablement. Dans notre cadre cela signifie que notre monde est le seul monde et que tout autre être postulé est un arrière-monde que fomente la subjectivité, et plus précisément la pensée paranoïaque polarisée par l'horizon du secret, du voilement. Enfin penser que le monde ait pu être précédé ou/et puisse être succédé par un état d'immobilité ou par un être autre que le monde revient aussi à projeter les dimensions temporelles de l'avant et de l'après sur un monde qui en soi échappe à la découpe temporelle, ne relève pas de la temporalité. Autrement dit, ces postulats naïfs ratent l'infinité d'un procès phusique surpuissant qui n'a ni début, ni fin ; ils ratent ce commencement inépuisable qui est le seul visage possible de l'éternité12. Le monde est cette origine qui se donne sous la forme d'une sempiternelle relance de la différenciation. Dans tous les cas, si le monde est un mouvement spatialisant éternel et prétemporel, on peut d'ores et déjà affirmer la priorité de l'espace sur le temps : la temporalité, qui ne surgira qu'avec le sujet (le vivant à vrai dire), présuppose la dynamique de cet espace archaïque.

\footnotetext{
${ }^{12} \mathrm{Il}$ resterait à montrer que si l'on saisit que l'éternité ne peut être que mobile alors on doit en finir avec la pensée qui associe les deux thèses complémentaires suivant lesquelles si l'immobilité est l'être de l'éternel - il suffit de citer l'être éléatique, l'eidos platonicien, l'acte pur aristotélicien, l'Un plotinien, le dieu de la théologie chrétienne, le principe logique de la nécessité de la contingence chez Meillassoux, etc. - alors la mobilité est l'être du temporel voire du passager - ainsi l'Élan vital bergsonien compris comme durée, la finitude des mouvements sublunaires chez Aristote, l'existence provisoire de notre univers en expansion pour l'astrophysique moderne, etc. La pensée de Barbaras amène à faire sécession avec cette tradition massive et à penser l'éternité propre d'un mouvement - certes il faudrait examiner de plus près certaines pensées qui, envisageant des figures d'éternité mobile, semblent s'excepter de cette tradition : ainsi l'héraclitéisme, l'atomisme, etc.
} 


\section{Origine événementielle du sujet}

Mais après avoir distingué le mouvement du monde de celui du sujet par une différence de puissance, il reste à éclairer la solution de continuité qui a été explicitement instaurée entre ces deux mouvements. À partir du mouvement surpuissant du monde rien ne permet de rendre compte du mouvement impuissant, de l'existence du sujet: la surpuissance ne peut que s'affirmer, elle ne peut que produire de l'étant en différenciant - la surpuissance ne peut s'affaiblir, s'interdire elle-même de produire sinon elle se nierait comme surpuissance. Cette " limitation interne à la surpuissance », cette "impossibilité au cœur de ce qui peut tout $» n^{\prime}$ est pas une impuissance : d'abord parce qu'être impuissant à se rendre impuissant $c^{\prime}$ est justement préserver sa pleine puissance; et ensuite cette impossibilité représente une limitation propre à toute essence en tant qu'elle ne peut contenir sa propre négation, à moins de se nier comme essence - l'impossibilité de se nier soi-même constitue l'essence de toute essence, ce qui en assure la consistance. Ainsi la surpuissance est étrangère à la notion théologique de toute-puissance divine qui recèle la capacité de se nier soi-même. En conséquence afin de rendre compte de la surrection du sujet il faut postuler l'événement d'un affaiblissement du monde. Si cet événement peut infléchir l'archi-mouvement du monde en son cœur c'est parce qu'il est aussi archaïque que lui et à ce titre mérite d'être nommé archiévénement. Cet archi-événement fragilisant n'est, certes, pas une destruction du monde - nous ne serions pas là pour en parler -, mais, pour deux raisons corrélatives majeures, il n'est pas non plus une force d'expulsion hors du monde. D'une part, parce que le sujet n'appartiendrait plus au monde ce qui nous installerait dans une contradiction aussi bien (méréo)logique que phénoménologique : exclu purement et simplement du monde, le sujet ne serait plus une partie du tout ultime et hors de ce tout formerait un nouveau tout étranger au tout du monde, un autre tout. Or, on l'a vu, la coexistence de deux tout absolus est une contradiction dans les termes. Si (méréo)logiquement il ne peut y avoir qu'un seul tout absolu alors aucune expulsion n'a la force de jeter hors du monde. Phénoménologiquement, si le sujet était univoquement séparé du monde, s'il ne lui appartenait tout simplement plus on perdrait l'a priori de la parenté ontologique, et on ferait du sujet un étranger, un tout autre que le monde. Dans ces conditions, le séparé serait d'une nature toute autre que ce dont il se sépare, le sujet serait une réalité sans commune mesure avec le monde de sorte qu'il serait un étant immobile et totalement impuissant. Or, cette détermination 
du sujet diverge complètement de celle que la phénoménologie a déduite - la partie détachée du monde par la lézarde événementiale demeure mouvement comme la queue détachée et vivante du lézard. D'autre part, et corrélativement, faute d'appartenir au monde, de pouvoir s'y tenir, le sujet serait incapable d'aspirer au monde situé audelà de toute distance. Absolument dépourvu de monde, on est bien en mal de saisir ce qui pourrait apparaître au sujet qui n'aurait dès lors plus rien d'un mouvement phénoménalisant. Ainsi, cet archi-événement correspond à un rien (de dynamique, et a fortiori de substantiel) affaiblissant qui s'immisce dans le procès mondain et le sépare de luimême au cœur de lui-même. Barbaras parle parfois de «chute $»^{13}$ : dans ce contexte métaphysique ${ }^{14}$ il ne faut évidemment pas prêter un sens axiologique et notamment judéo-chrétien à ce terme, et par ricochet à l'événement qu'il qualifie, puisqu'il s'agit seulement de décrire le surgissement d'une chute de puissance au sein de la surpuissance, la surrection interne d'un néant qui fait choir le monde de lui-même ${ }^{15}$.

Il reste qu'en thématisant un tel archi-événement, Barbaras aménage le champ pour une nouvelle époque métaphysique qui bat en brèche le principe philosophique par excellence qu'est le principe de raison suffisante. Tout d'abord, ce principe somme le philosophe de débusquer les causes ou les raisons de chaque chose. Or, dans le contexte cosmologique établi, à la question réclamant de savoir quelle est la raison ou la cause pour ou par laquelle le sujet (le mouvement

\footnotetext{
${ }^{13}$ DM, p. 247.

${ }^{14}$ Ce contexte où s'installe la réflexion concernant l'archi-événement est éminemment métaphysique pour un certain nombre de raisons que Barbaras développe longuement dans Dynamique de la manifestation, troisième partie. Mais succinctement on peut faire remarquer deux choses qui légitiment l'utilisation spontanée de ce terme : d'une part, que l'événement ne relève en rien de l'être, de la phusis et en ce sens l'excède, la transcende - sans pour autant que ce rien ne constitue une quelconque positivité - de sorte que le discours de l'archi-événement peut être dit «métaphysique » au sens traditionnel en ce qu'il thématise une réalité « au-delà de la phusis ». D'autre part, ce discours de l'archi-événement se déploie nécessairement après la cosmologie, le discours sur la phusis : il s'intitule donc naturellement « métaphysique » car en tant que discours « aprèsla-physique » il en corrobore pleinement le sens littéral.

${ }^{15} S^{\prime}$ il ne faut pas attribuer un sens axiologique au terme de « chute » dans la philosophie de l'événement que développe Barbaras, il faut tout de même remarquer que l'homonymie avec l'événement de la Genèse biblique est significative. Encore une fois, il faudrait ontologiser le mythe de la Genèse en ôtant les apprêts axiologiques du récit et en ressaisissant ses intuitions révélatrices sous-jacentes («la chute », « l'expulsion », « l'arbre de vie », etc.). Car alors apparaîtrait la vérité ontologico-métaphysique en tant que telle, $c^{\prime}$ est-à-dire, cette vérité plus profonde que celle de la morale et ou celle des faits. Dans les Promenades du promeneur solitaire, Rousseau inféodait la vérité des faits à la vérité morale - « cent fois plus respectable » - et prônait ou non la fiction, l'invention de faits en fonction de sa destination morale. Il faudrait en quelque sorte reproduire, reconduire ce geste à un niveau supérieur pour neutraliser le niveau factuel aussi bien que moral, et prôner telle ou telle histoire en fonction de la profondeur ontologico-métaphysique de l'intuition qu'il met en scène.
} 
impuissant) apparaît, la réponse ne peut être que «pour aucune raison ». C'est l'impossibilité même d'une réponse concluante, le repérage d'une impasse empêchant de faire droit à l'horizon d'attente de cette question, qui enjoint le penseur à postuler un surgissement sans cause ni raison, à déduire un événement, c'est-à-dire une réalité métaphysique qui échappe à la fois à l'ordre de l'être, du monde et aux principes qui semblent le régir. Cette «absence de réponse » est donc ce qui permet de répondre par l'absence de raison : le sujet est bien le sans raison par excellence et l'interrogation métaphysique de son origine rend caduc le commandement du principe de raison. Le sujet est cette réalité qui peut raisonnablement assigner des raisons ou des causes à toute réalité, fors à la sienne (et à celle du tout lui-même). Cette métaphysique aura donc pour objet ce rien sans cause, ni raison, ce néant événementiel et pourra se caractériser comme mêdenologie en tant que «mêden » désigne bien « rien » en grec. Conséquemment à cette ruade contre l'exigence traditionnelle de la philosophie, cette mêdenologie propose une toute autre question que celle présupposée par le principe de raison suffisante. En effet, traditionnellement, le principe de raison suffisante présuppose la question directrice suivante : «Pourquoi y a-t-il quelque chose plutôt que rien?». Dans L'Évolution créatrice, Bergson a sans doute été le premier à désigner le piège substantialiste que tend cette question à tous ceux qui l'adoptent comme fil conducteur de leurs recherches philosophiques : en effet, penser l'être en le profilant sur fond de néant amène nécessairement à une conception de l'être comme pure positivité, substance et corrélativement à lui refuser la moindre négativité. C'est pourquoi selon une tradition parménidienne - qui passe, et ne s'arrête pas, par Bergson luimême en tant qu'il affirme un devenir, une durée encore substantielle - l'être a été caractérisé substantiellement, statiquement et non dynamiquement. Et il ne suffit pas d'inverser cette question directrice pour clore la philosophie traditionnelle et sa perspective substantialiste, statique. En atteste la dramaturgie métaphysique que développe Sartre dans L'Être et le Néant : tentant de rendre compte de la surrection du sujet, du pour-soi par un en-soi visant à se fonder lui-même, Sartre renverse en quelque sorte le questionnement : "pourquoi y a-t-il (le) rien plutôt que (seulement) l'être ? » Certes, Sartre repère bien qu'il y a de la négativité dans l'être - le sujet est cette poche négative - et donc que la question ne peut formuler une alternative tranchée entre le positif et le négatif. Même si ce renversement refuse donc l'exclusivité de l'être ou du néant, Sartre s'obstine à penser la négativité subjective sur fond de pure positivité, de plénitude ontologique, d'en-soi et est naturellement amené à saisir la négativité du sujet comme pur néant, 
néant substantiel, trou. Ainsi, bien qu'il inverse la question inaugurale et veut rendre compte d'une certaine perméabilité entre l'être et la négativité, il $\mathrm{n}^{\prime}$ arrive jamais à sortir du substantialisme traditionnel : néant soit-il, le sujet n'en reste pas moins réifié. À aucun moment Sartre n'essaye de rompre l'alternative massive de l'être et du néant, et sa philosophie est une «maquette abstraite » qui les tient juxtaposés : il ne peut donc jamais penser un être infiltré de négativité, un être dynamique comme Patočka puis Barbaras se proposent de le faire. Sartre est bien un des derniers avatars, sinon le dernier, de l'ontologie traditionnelle. Si la phénoménologie et la cosmologie barbarassiennes neutralisent déjà l'alternative abstraite de l'être et du néant, la métaphysique mêdenologique pose une toute autre question car le néant qu'elle repère et qui affecte l'être en son cœur n'a rien de commun avec un néant substantiel, ni avec la négativité de tout mouvement (en tant qu'elle recèle encore la positivité de déposer un substrat). Il s'agit, on l'a vu, d'un néant événementiel, d'une absolue négativité. Ainsi, l'abord événementiel du néant ne se contente pas d'inverser et de contester l'illustre formule, elle annihile radicalement le "pourquoi », la marotte de la raison et de la cause - l'événement étant du côté de la gratuité imprévisible, absolument sans cause ni raison ${ }^{16}$. Par conséquent cette mêdenologie inédite suppose et pose la question inaugurale suivante : «Comment y a-t-il (le) rien plutôt que (seulement) quelque chose? » Cette question directrice, dont il reste à mesurer précisément la nouveauté métaphysique, somme, en tous les cas, de saisir comment la néantité de l'archi-événement peut surgir au cœur du mouvement du monde et l'affecter. Autrement dit, puisque rien n'échappe au tout absolu qu'est le monde, comment le rien événementiel $n^{\prime}$ échappe pas au tout sans pour autant en relever ou $s^{\prime} y$ réduire? Et comment par le surgissement de ce rien, le tout peut-il vaciller? De quelle façon ce rien du tout pousse le tout à s'échapper de lui-même?

À ce stade de la restitution, on doit déjà reconnaître que la phénoménologie de Barbaras inaugure une perspective résolument spatialiste. En effet, il est déjà clair qu'au sein de cette pensée l'articulation de l'espace et du temps ne met pas en jeu un conditionnement réciproque, mais implique bien plutôt la priorité de l'espace sur le temps puisque la phusis éternelle est un espace inchoatif et ductile seulement pré-temporel. En tant que néant absolu, l'archi-événement

\footnotetext{
${ }^{16}$ Les atomistes épicuriens ont sans doute ouvert la brèche d'une telle métaphysique du néant événementiel - même si le néant substantiel, éléatique du vide subsiste - en thématisant la déviation/déclinaison événementielle des atomes, le clinamen.
} 
ne pourra lui-même représenter aucune réalité spatiale ou temporelle, mais il devra s'entendre comme une instance scissionnante permettant $l^{\prime}$ avènement $d$ 'autres espèces d'espaces et de la temporalité en tant que telle. Bien entendu, toute la difficulté résidera dans l'exigence à penser la dérivation, indirecte fût-elle, du temps à partir de l'espace archaïque. 